\title{
Potentiometrischer $\mathrm{CO}_{2}$-Sensor in Dickschichttechnologie zur Atemgasanalyse
}

Sven Wiegärtner ${ }^{1}$, Gunter Hagen ${ }^{1}$, Jaroslaw Kita ${ }^{1}$, Manuel Seufert ${ }^{2}$, Eckard Glaser ${ }^{2}$, Kerstin Grimmel $^{2}$, Christa Schmaus ${ }^{3}$, André Kießig ${ }^{3}$, Armin Bolz ${ }^{2}$, Ralf Moos ${ }^{1}$.

${ }^{1}$ Lehrstuhl für Funktionsmaterialien, Universität Bayreuth, 95440 Bayreuth, Deutschland

${ }^{2}$ Corscience GmbH \& Co. KG, 91052 Erlangen, Deutschland

${ }^{3}$ Siegert electronic GmbH, 90556 Cadolzburg, Deutschland

\section{Zusammenfassung}

In diesem Beitrag wird ein elektrochemischer Sensor des Typs $\mathrm{CO}_{2}, \mathrm{O}_{2}, \mathrm{Au}, \mathrm{Li}_{2} \mathrm{CO}_{3}-\mathrm{BaCO}_{3}|\mathrm{Nasicon}|$ $\mathrm{Na}_{2} \mathrm{Ti}_{6} \mathrm{O}_{13}-\mathrm{TiO}_{2}, \mathrm{Au}, \mathrm{O}_{2}, \mathrm{CO}_{2}$, der in kostengünstiger Dickschichttechnik reproduzierbar hergestellt wird, beschrieben. Eine Miniaturisierung des Sensors und die Integration eines Heizelements auf der Rückseite des Sensors erlaubt es, diesen eigenständig bei der benötigten Betriebstemperatur von $525{ }^{\circ} \mathrm{C}$ zu betreiben. Dabei hängt das Messsignals vom $\mathrm{CO}_{2}$-Partialdruck der zu messenden Umgebung ab und gehorcht der Nernst-Gleichung. Der Sensor kann zur Atemgasanalyse eingesetzt werden.

\section{Motivation}

Die Analyse bzw. die Überwachung der menschlichen Atemluft ist für einige Anwendungen, wie z.B. für die Leistungsdiagnostik oder die Beatmungskontrolle, unverzichtbar [1]. Jedoch stellt diese zahlreiche hohe Anforderungen an den Sensor, wie ein schnelles Ansprechverhalten, eine hohe Selektivität und eine ausreichende Langzeitstabilität. Nach [2] erfüllen vor allem potentiometrische Sensoren diese Anforderungen.

Potentiometrische Sensoren wurden in Form einer elektrochemische Zelle des Typs $\mathrm{CO}_{2}, \mathrm{O}_{2}, \mathrm{Au}, \mathrm{Li}_{2} \mathrm{CO}_{3}$ $\mathrm{BaCO}_{3} \mid$ Nasicon $\mid \mathrm{Na}_{2} \mathrm{Ti}_{6} \mathrm{O}_{13}-\mathrm{TiO}_{2}, \mathrm{Au}, \mathrm{O}_{2}, \mathrm{CO}_{2}$ in Anlehnung an [3] und [4] komplett in Dickschichttechnik hergestellt. Anstelle von $\mathrm{Na}_{2} \mathrm{CO}_{3}$ wurde wegen der geringeren Querempfindlichkeit von $\mathrm{Li}_{2} \mathrm{CO}_{3}$ auf Wasserdampf [5] eine eutektische Mischung (Molverhältnis 2:1) aus $\mathrm{Li}_{2} \mathrm{CO}_{3}$ und $\mathrm{BaCO}_{3}$ als Arbeitselektrodenmaterial verwendet. Dieses Sensorsystem ist thermodynamisch eindeutig definiert [3] und gehorcht somit der Nernst-Gleichung (1) mit dem Standardpotenzial $E_{0}$, der allgemeine Gaskonstante $R$, der Sensortemperatur $T$, der Elektronenübergangszahl $n$, der Faraday-Konstante $F$, dem Umgebungsdruck $p_{0}$ und dem zu detektierendem $\mathrm{CO}_{2}$-Partialdruck

$$
U_{\text {Sensor }}=E_{0}-\frac{R \cdot T}{n \cdot F} \cdot \ln \left(\frac{p\left(\mathrm{CO}_{2}\right)}{p_{0}}\right)
$$

Die Herstellung im Mehrfachnutzen erlaubt die Realisierung kostengünstiger Bauteile. Auf der Rückseite der Sensoren wurden - ebenfalls im Siebdruckverfahren - Platinstrukturen aufgebracht, die zum Beheizen der Sensoren dienen. Über den temperaturabhängigen Widerstand $(T K R=3660 \mathrm{ppm} / \mathrm{K}) \mathrm{kann}$ der jeweilige Sensor geregelt bei der optimalen Betriebstemperatur von $525^{\circ} \mathrm{C}$ betrieben werden.

\section{Experimentelles}

\subsection{Sensoraufbau}

Die hier entwickelten potentiometrischen $\mathrm{CO}_{2}$-Sensoren wurden in Ahnlehnung an [4] komplett in Dickschichttechnik hergestellt. In Bild 1 wird ein Querschnitt des Sensors gezeigt. Bei der Herstellung der Sensoren dient eine Seite des Aluminiumoxidsubstrates als Träger der Sensorfunktionsschichten (im Folgenden: Sensorseite). Hierzu wird zunächst die Elektrolytschicht - Nasicon - auf die Sensorseite im Siebdruckverfahren aufgebracht. Danach wird auf einer Seite der Elektrolytschicht das Referenzelektroden- 
material $\mathrm{Na}_{2} \mathrm{Ti}_{6} \mathrm{O}_{13} \mid \mathrm{TiO}_{2}$ gedruckt. In einem weiteren Druckschritt werden zwei Goldgitterelektroden angebracht, um letztendlich als letzte Schicht das Arbeitselektrodenmaterial $\mathrm{Li}_{2} \mathrm{CO}_{3} \mid \mathrm{BaCO}_{3}$ zu drucken.

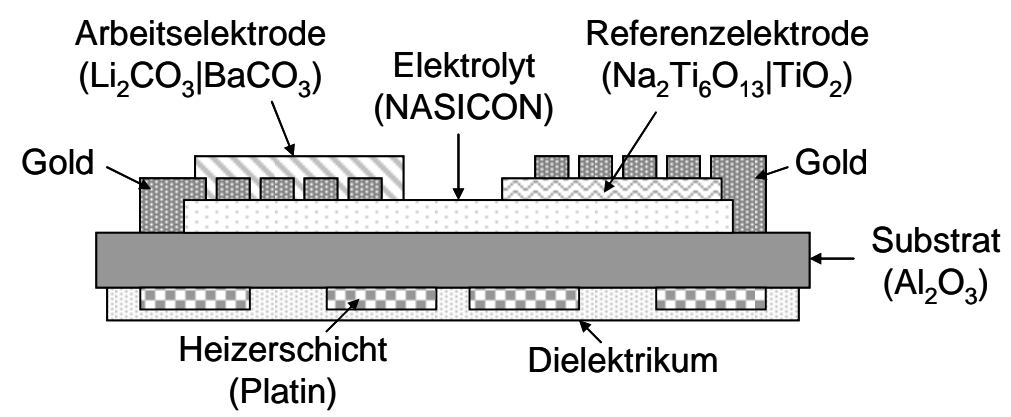

Bild 1 Querschnitt durch die Sensorschichten. Auf der Oberseite des Substrates sind die Funktionsmaterialien des Sensorelements angebracht. Auf der Unterseite des Substrates ist das Heizelement lokalisiert.

Auf die Rückseite des Aluminiumoxidsubstrates (im Folgenden: Heizerseite) wird ein Heizelement angebracht. Dazu wird eine Platinstruktur auf das Substrat angebracht, die als Heizelement dient. Zur besseren Langzeitstabilität der Heizerschicht wird diese noch mit einer Dielektrikumsschicht versehen. Zur Stromversorgung des Heizelements werden noch Goldzuleitungen siebgedruckt.

Aufgrund der planaren Heizerstruktur auf der Rückseite des Sensors ist es möglich, diesen als eigenständiges Gerät bei der benötigten Betriebstemperatur zu betreiben. Zusätzlich kann mithilfe des bekannten temperaturabhängigen Widerstands der Platin-Heizerschicht, die Sensortemperatur geregelt werden. Somit ist es möglich den vorgestellten $\mathrm{CO}_{2}$-Sensor für verschiedene Anwendungen zu einzusetzen. Hier soll die Überwachung menschlicher Atemluft vorgestellt werden.

\subsection{Messungen}

Um den Sensor als eigenständigen Sensor zu betreiben, sind Voruntersuchungen zur Charakterisierung des Sensor- und des Heizelements nötig. Dazu wurden sowohl die Kennlinien des Sensorelements, als auch die Abhängigkeit des Heizerwiderstands von der Temperatur untersucht. Die Sensoren wurden mithilfe eines Probenträgers in einem Ofen platziert und auf die benötigte Betriebstemperatur von $525{ }^{\circ} \mathrm{C}$ beheizt. Zusätzlich wurden sie mit Testgas beaufschlagt, welches aus $20 \% \mathrm{O}_{2}$, variierenden $\mathrm{CO}_{2}$-Konzentrationen (von 0,4 \% $\mathrm{CO}_{2}$ bis zu 3,9\% $\mathrm{CO}_{2}$ ) und $\mathrm{N}_{2}$ besteht. Während der kompletten Messungen wurde sowohl die Sensorspannung als auch der Heizerwiderstand gemessen, um jeweils die Kennlinien des Sensors bzw. des Heizers zu ermitteln.

In einem zweiten Schritt wurde der Sensor mit dem integrierten Heizelement selbstbeheizt unter realitätsnahen Bedingungen getestet. Hierzu wurde der Sensor in einen tubusartigen Aufbau eingebaut. Die Sensortemperatur wurde über den bekannten Platinwiderstand eingestellt und während des Betriebs auf diesen Widerstand geregelt. Als Testgas diente die Atemluft einer Testperson, die durch den Aufbau ein- und ausatmete. Anhand der gemessenen Sensorspannung während des Versuchs und der bereits ermittelten Sensorkennlinie kann auf die $\mathrm{CO}_{2}$-Konzentration geschlossen werden.

\section{Ergebnisse und Diskussion}

\subsection{Charakterisierung des Sensorelements}

Bild 2 zeigt das Sensorsignal einer Messung zur Charakterisierung des Sensorsignals. Wie bereits erwähnt wurde der Sensor dabei in einem Ofen mit verschiedenen $\mathrm{CO}_{2}$-Konzentrationen, ausgehend von 0,4 \% bis zu 3,9\% $\mathrm{CO}_{2}$ beaufschlagt und die Sensorspannung gemessen. Es ist deutlich zu erkennen, dass - wie theoretisch erwartet - bei steigenden $\mathrm{CO}_{2}$-Gehalten das Sensorsignal $U_{\text {Sensor }}$ abnimmt. 


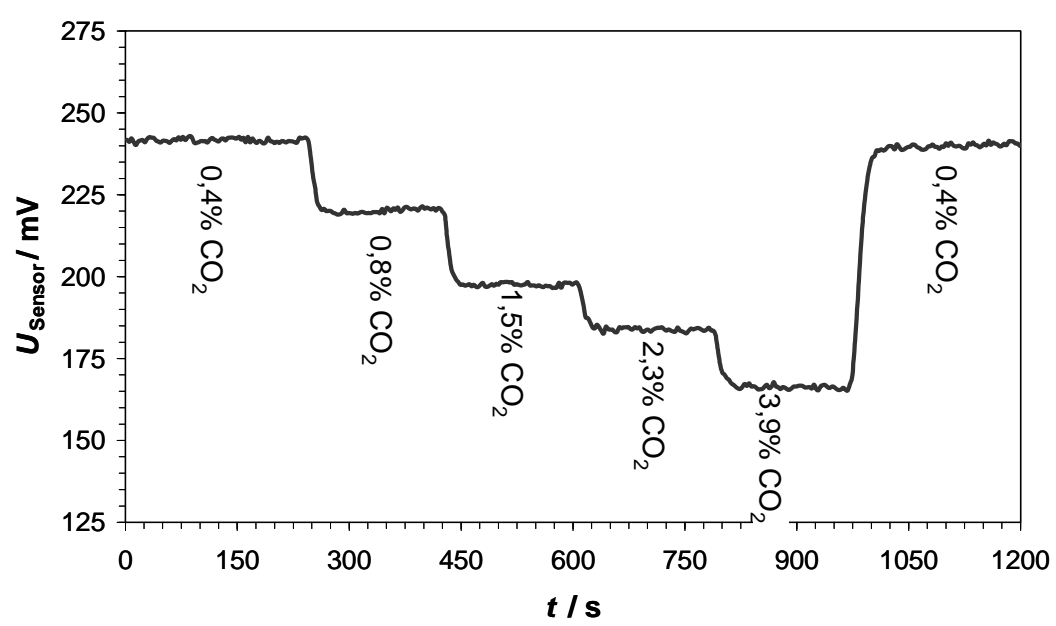

Bild 2 Gemessenes Spannungssignal eines planaren potentiometrischen $\mathrm{CO}_{2}$-Sensors mit $\mathrm{Li}_{2} \mathrm{CO}_{3} \mid \mathrm{BaCO}_{3}$ als Arbeitselektrodenmaterial während variierender $\mathrm{CO}_{2}$-Gaskonzentrationen.

Zur Erstellung der Sensorkennlinien wurden die jeweiligen Spannungswerte am Ende eines Plateaus gegen die jeweilige Konzentration aufgetragen. In Bild 3 sind die auf diesem Wege ermittelten Kennlinien von fünf Sensoren eines Substrates (es werden 40 Sensoren pro Substrat hergestellt) dargestellt. In Bild 3a sind die Kennlinien in einer halblogarithmischen Darstellung, wie sie aus wissenschaftlicher Sicht interessant sind, aufgetragen. Hingegen ist die lineare Darstellung der Kennlinien, wie in Bild 3b, für eine anwendungsorientierte Darstellung geeignet.
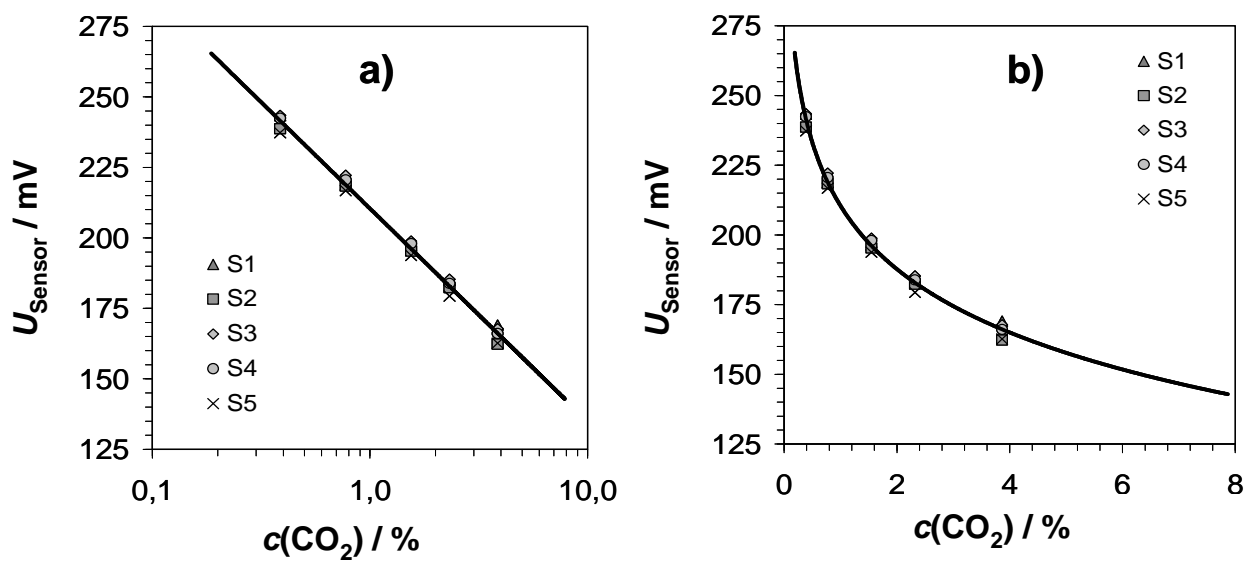

Bild 3 Kennlinien von fünf getesteten Sensoren eines Substrates. a) halblogarithmische Darstellung b) lineare Darstellung

In Bild 3a wird gezeigt, dass die Sensoren reproduzierbar herstellbar sind. Alle getesteten fünf Sensoren eines Substrates weisen eine gleiche Steigung von $75 \mathrm{mV} /$ Dekade $\mathrm{CO}_{2}$-Konzentration auf und besitzen alle einen gleichen Offset.

In Ahnlehnung an [2] und [3] wird die halblogarithmische Darstellung der Sensorkennlinie herangezogen, um das Sensorverhalten mit Bezug auf die Nernst-Gleichung (1) zu charakterisieren. Anhand der Steigung $m$ der Kennlinie kann die Elektronenübergangszahl $n$ nach folgender Formel (2) berechnet werden.

$$
U_{\text {Sensor }}=E_{0}-\frac{R \cdot T}{n \cdot F} \cdot \ln \left(\frac{p\left(\mathrm{CO}_{2}\right)}{p_{0}}\right)=E_{0}-\underbrace{\frac{R \cdot T \cdot \ln 10}{n \cdot F}}_{m} \log \left(\frac{p\left(\mathrm{CO}_{2}\right)}{p_{0}}\right)
$$

Aufgrund der elektrochemischen Reaktionen, die an den Sensorelektroden ablaufen, wird theoretisch eine Elektronenübergangszahl $n=2$ erwartet. Bei den hier getesteten Sensoren liegt die Elektronenübergangszahl mit $n=2,2 \pm 0,1$ nahe dem erwarteten theoretischen Wert. Dies bestätigt, dass die Sensoren mit der Theorie übereinstimmen. 


\subsection{Messung unter realen Bedingungen mit Atemluft}

In einem ersten Funktionstest der Sensoren wurde ein charakterisierter Sensor in einen tubusartigen Aufbau eingebaut und über das integrierte Heizelement auf Betriebstemperatur $\left(525^{\circ} \mathrm{C}\right)$ gebracht. Durch den Aufbau atmete eine Person ein und aus. Während des Versuchs wurde die Temperatur des Sensorelements über den Widerstand des Heizelements geregelt. Mithilfe der zuvor ermittelten Sensorkennlinie kann mit der gemessenen Sensorspannung die vorherrschende $\mathrm{CO}_{2}$-Konzentration in der Atemluft bestimmt werden. Bild 3a zeigt die gemessene Konzentration während mehreren Atemzyklen der Testperson.

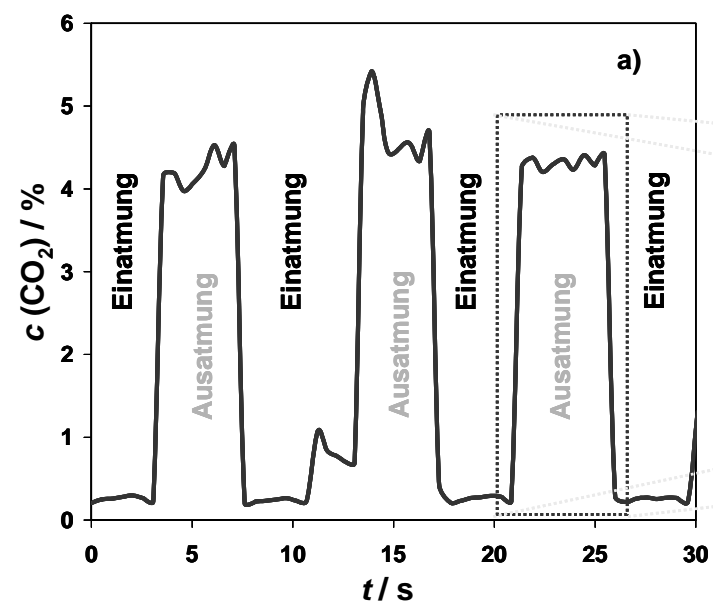

b)

Bild 3 a) Gemessene $\mathrm{CO}_{2}$-Konzentration während mehrerer Atemzyklen einer Testperson b) Vergleich der gemessenen $\mathrm{CO}_{2}$-Konzentration (gestrichelt) mit einem Kapnogramm aus der Literatur (Linie) (vgl. [6])

Während den Einatmungsphasen wurden wie erwartet nahezu $0 \% \mathrm{CO}_{2}$ gemessen, wohingegen während des Ausatmens zwischen 4\% und 5\% $\mathrm{CO}_{2}$ detektiert wurden. In Bild 3b wird der umrahmte Teil aus Bild 3a mit einem Kapnogramm aus der Literatur [6] verglichen. Hierbei wird deutlich, dass der Anstieg und der Abfall der $\mathrm{CO}_{2}$-Konzentration aufgrund der geringen Ansprechzeit der Sensoren dem Erwarteten entsprechen. Auch am Ende der Plateauphase während der Ausatmung stimmen Praxis mit Theorie überein. Lediglich am Anfang der Plateauphase liegt das Sensorsignal oberhalb des typischen Kapnogramms, was in einer noch zu verbessernden Dynamik der Temperaturreglung begründet ist. Dies führt zu einem leichten Überschwingen der Sensortemperatur, wie mithilfe eines Sensors, welcher an der Oberseite einen Temperatursensor anstatt der gassensitiven Schicht enthielt, gezeigt wurde. Hieran wird in Zukunft gearbeitet werden.

\section{Fazit}

Es wurde gezeigt, dass sich potentiometrische $\mathrm{CO}_{2}$-Sensoren mit integriertem Heizelement reproduzierbar herstellen lassen. Aufgrund der guten Ansprechzeit dieser Sensoren und dem Erfüllen der theoretischen Erwartungen eignen sich diese Sensoren zur Atemluftüberwachung.

\section{Danksagung}

Dieses Projekt wird von der Bayerischen Forschungsstiftung (AZ-879-09) gefördert. Vielen Dank.

\section{Literatur}

[1] D. Biarent, R. Bingham, C. Eich, J. López-Herce, I. Maconochie, A. Rodriguez-Núnez, T. Rajka, and D. Zideman, "European resuscitation council guidelines for resuscitation 2010, Abschnitt 6. Paediatric life support," Resuscitation, 81 (2010) 1364-1388.

[2] P.Pasierb, M. Rekas, "Solid-state potentiometric gas sensors - current status and future trends,” Journal of Solid State Electrochemistry, 13 (2009) 3-25.

[3] M. Holzinger, J. Maier, and W. Sitte, "Fast $\mathrm{CO}_{2}$-selective potentiomteric sensor with open reference electrode," Solid State Ionics, 86-88 (1996) 1055-1062.

[4] K. Sahner, A. Schulz, J. Kita, R. Merkle, J. Maier, and R. Moos, “ $\mathrm{CO}_{2}$ selective potentiometric sensor in thick-film technology," Sensors, 8 (2008) 4774-4785.

[5] S. Yao, Y. Shimizu, N. Miura, and N. Yamazoe, "Solid electrolyte carbon dioxide sensor using sodium ionic conductor and lithium carbonate-based auxiliary phase," Applied Physics A, 57 (1993) 25-29.

[6] J.E. Thompson, M.E. Jaffre, “Capnographic Waveforms in the Mechanically Ventilated Patient,” Respiratory Care, 50 (2005) 100-109. 